Article

\title{
Detailed Investigations on the Oil Flow in Dip-Lubricated Gearboxes by the Finite Volume CFD Method ${ }^{\dagger}$
}

\author{
Hua Liu *(1), Thomas Jurkschat ${ }^{(0)}$, Thomas Lohner ${ }^{(0)}$ and Karsten Stahl \\ Gear Research Centre (FZG), Technical University of Munich, Boltzmannstraße 15, 85748 Garching b. München, \\ Germany; thomas.jurkschat@web.de (T.J.); lohner@fzg.mw.tum.de (T.L.); stahl@fzg.mw.tum.de (K.S.) \\ * Corresponding author: liu@fzg.mw.tum.de \\ † This paper is an extended version of our paper published in 6th World Tribology Congress, Beijing, China.
}

Received: 9 February 2018; Accepted: 2 May 2018; Published: 9 May 2018

\begin{abstract}
Three main concerns are in the focus of the development of geared transmissions nowadays: load carrying capacity, noise-vibration-harshness (NVH) behavior, and efficiency. Increasing the efficiency of modern gearboxes contributes significantly to the reduction of energy consumption and the saving of resources. Gearboxes are frequently designed conservatively with an oversupply of oil to guarantee operational reliability. An oversupply of oil results in an unnecessarily high amount of oil kept in motion and to high no-load losses. Detailed information on the oil distribution in the early design stages of gearboxes would help to optimize the lubrication and to increase the efficiency. Thereby, CFD (computational fluid dynamics) methods offer a very flexible way to visualize the oil flow inside gearboxes with much fewer restrictions compared to measurements with transparent gearbox designs. In this study, a verified CFD model based on the finite volume method is used to investigate the oil flow in a single-stage gearbox. Different oil viscosities and circumferential speeds are considered. The investigations focus on the oil flow. The gear churning loss, as part of the no-load loss, is additionally considered. Experimental validation is obtained by high-speed camera recordings and measurements at the FZG no-load power loss test rig. The results show very strong agreement between simulation and measurement. The results show that CFD simulations can visualize the oil flow behavior with a very high degree of detail.
\end{abstract}

Keywords: gear efficiency; CFD simulation; oil distribution; oil flow; churning losses

\section{Introduction}

Improving the efficiency of geared transmissions is one of the most significant goals in the development of current drive systems. Various investigations [1-4] showed that the no-load gear losses $\mathrm{P}_{\mathrm{LG} 0}$ often hold a remarkable percentage of the overall power loss and, thus, play a significant role in the development of a gearbox.

The no-load losses of dip-lubricated gears can be categorized based on their mechanism of formation into churning, squeezing and windage (Mauz [2]):

$$
\mathrm{P}_{\mathrm{LG} 0}=\mathrm{P}_{\mathrm{LG} 0, \mathrm{C}}+\mathrm{P}_{\mathrm{LG} 0, \mathrm{~S}}+\mathrm{P}_{\mathrm{LG} 0, \mathrm{~W}}
$$

The loss caused by gears dipping and circulating in the oil sump is identified as churning loss $\mathrm{P}_{\mathrm{LG} 0, \mathrm{C}}$. The squeezing loss $\mathrm{P}_{\mathrm{LG}, \mathrm{S}}$ originates from the oil squeezed out of the tooth gaps at the gear meshing zone. Thereby, oil is displaced in the rolling and axial directions. One part is caused by the displacement of the oil film on the tooth flank in the rolling direction and the second part by the axial squeezing of excessive oil out of the tooth gap. The windage loss $P_{\mathrm{LG}, W}$ is caused by the secondary 
medium, generally air. The hydraulic loss generally includes the churning and squeezing loss in the case of dip-lubrication.

Figure 1 exemplifies the churning, squeezing and windage loss in a dip-lubricated gearbox based on CFD simulation results obtained by the model of Liu et al. [5].

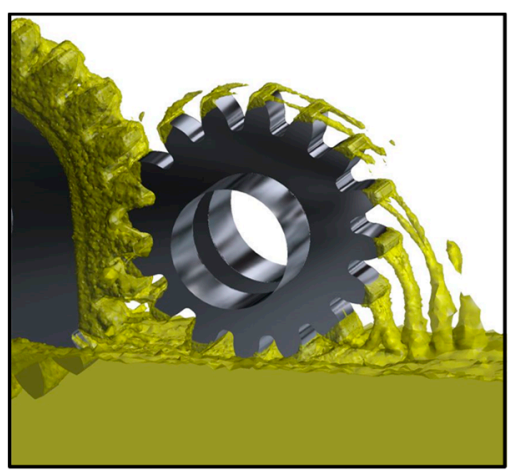

Churning

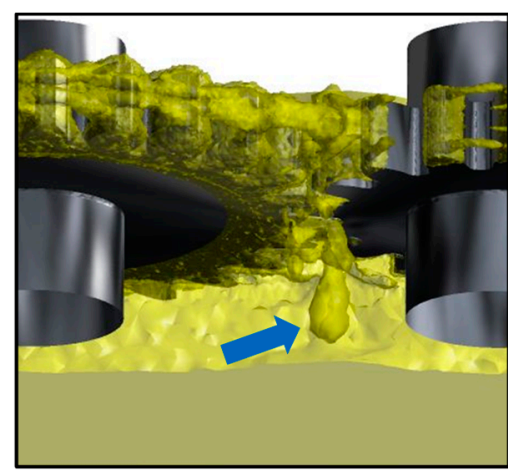

Squeezing

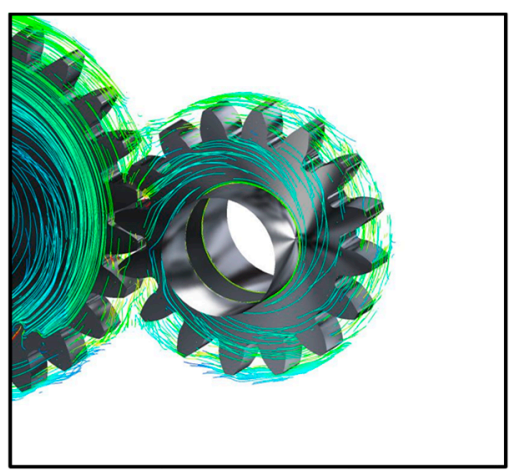

Windage

Figure 1. Illustration of different kinds of no-load losses based on CFD simulation (oil distribution plot for churning and squeezing loss and velocity plot for windage loss).

The no-load gear power loss and the oil flow in gearboxes have been the focus of much research for many years. Mauz [2] focused on the churning and impulse power losses of spur gears for various operating conditions and derived a set of empirical equations with influencing parameters, such as circumferential speed and oil viscosity. Doleschel [6] confirmed the significant influence of oil viscosity and identified the oil fill level as one of the main influencing parameters on the no-load power loss. In addition to oil viscosity and density, Leprince et al. [7] pointed out the importance of oil properties related to oil aeration. Otto [8] generally emphasizes the influence of the oil flow on the no-load gear power loss.

In view of increasing computing power in recent years, CFD methods have gradually been applied to investigate the oil flow and no-load loss of geared transmissions. An overview of conducted studies was already given by the authors in [5]. Therefore, the following summary of the literature concentrates mainly on additional studies not mentioned in [5].

By using a two-dimensional finite volume (FV)-based CFD model, Li et al. [9] analyzed the influence of rotational speed, immersion depth and oil viscosity on the oil distribution of a single-stage dip-lubricated gearbox. They asserted that the turbulence of the oil flow increases with the rotational speed and the oil fill level. Li et al. [9] concluded that a three-dimensional CFD simulation model is required in order to gain detailed information on the oil flow inside gearboxes.

Jafarian [10] set up a three-dimensional FV-based CFD model with an overset meshing technique to investigate the oil distribution of a single stage dip-lubricated gearbox. The velocity field of the flow pattern was compared between the simulation results and particle image velocimetry (PIV) measurement results. A good level of agreement was found.

Klier and Berger [11] presented a work modeling a gear pair with an overset mesh method capable of representing the meshing of two gears. The FV-based CFD model gives a plausible prediction of the oil flow inside a single-stage dip-lubricated gearbox. The simulation results were not validated due to a lack of experimental data.

Concli et al. [1,12-16] conducted several studies on the CFD simulation of oil flow and churning power loss of spur gears and of planetary gears by using the FV method. It is pointed out that CFD methods provide a reliable tool for expanding the knowledge on oil flow and hydraulic losses in gearboxes. In the framework of their recent studies [16], a very time-efficient mesh-handling technique was applied. With this approach, the hydraulic losses of a fully oil-filled gearbox were simulated. The results showed good accordance with experimental data. 
By applying an FV-based CFD model, Burberi et al. [17] investigated the gear power loss of a single gear and a spur gear pair in a fully oil-filled gearbox for underwater operations. Using both the immersed solid and the dynamic meshing method, they conducted simulations to calculate the velocity field, the pressure, as well as the gear power losses. The results were compared to experimental measurements conducted by Concli et al. [18]. They concluded that the dynamic mesh method gives a better prediction of the gear power losses especially for a gear pair.

Liu et al. [5] applied a three-dimensional FV-based CFD model to investigate the oil flow and the churning power loss of a single-stage dip-lubricated gearbox. The simulation results in terms of oil distribution and churning loss torque are in very good agreement with high-speed camera recordings and torque measurements, respectively. It was concluded that the FV method is very suitable for determining the oil flow and the churning power loss in geared transmissions.

At the end of the 1970s, a particle-based approach, known as smoothed particle hydrodynamics (SPH), was introduced by Lucy et al. [19]. Thereby, the entire fluid domain is filled by particles carrying conservative fluid parameters. This method does not require a mesh in a classical sense. From a numerical point of view, the SPH method offers great potential for simulating oil flow in geared transmissions due to its GPU-based generic algorithm, which is very effective in computational parallelization. This provides an essential advantage in terms of computational performance over classical FV methods [20].

Iino et al. [21] used the moving particle semi-implicit (MPS) method to simulate the oil flow and the churning loss of a dip-lubricated spur gear pair. The results with regard to the oil distribution are plausible from a physical point of view. The results in terms of the churning loss were compared to the experimental measurements and show large deviations.

Liu et al. [20] applied a CFD simulation model based on the smooth particle hydrodynamics (SPH) method to investigate the oil flow and the churning power loss of a single-stage dip-lubricated gearbox. The SPH method could not reproduce the effect of the oil viscosity on the churning loss as shown in [5]. It is pointed out that further optimizations on current SPH codes are required for good predictions of no-load gear losses in geared transmissions.

By applying an SPH-based CFD model to a realistic automatic geared transmission model, Groenenboom et al. [22] were able to give information on the oil flow of a system consisting of 15 gears in two gearboxes. Despite a lack of experimental validation, the SPH method shows great potential for predicting the oil flow inside complex transmission systems due to its strongly reduced computing time compared to the FV method.

Through the years, various CFD simulations have been conducted analyzing the oil flow and no-load loss of dip-lubricated geared transmissions. Although SPH methods show great potential, FV methods provide currently better predictions [20,22]. Only a few works provide detailed validation of the simulated oil distribution with experimental measurements. Therefore, the FZG no-load power loss test rig has been reconstructed with a transparent cover made of acryl glass, allowing a detailed view inside the gearbox by a high-speed camera. The applied numerical FV-based CFD model is a continuing work of Liu et al. [5]. Different to [5], the current work focuses on the oil flow and its validation by high-speed camera recordings and, additionally, includes the influence of the lubricant. This also required model adjustments and different operating conditions. Preliminary results of this study were partly presented at a technical session at the 6th World Tribology Congress in Beijing in 2017 [23].

\section{Object of Investigation and Operating Conditions}

The object of investigation of this study is the FZG no-load power loss test rig. It features exactly the same test gear box as the FZG efficiency gear test rig used by the authors in $[18,19]$. The FZG no-load power loss test rig was specially designed for the measurement of no-load losses with high accuracy. In contrast to the FZG efficiency test rig, the FZG no-load power loss test rig can be driven at much higher speeds and is not based on the principle of power circulation. 


\subsection{FZG No-Load Power Loss Test Rig}

The mechanical layout and the test gearbox of the FZG no-load power loss test rig are shown in Figure 2. The main parts are the electric engine, the intermediate gearbox, the loss torque meter, and the test gearbox.
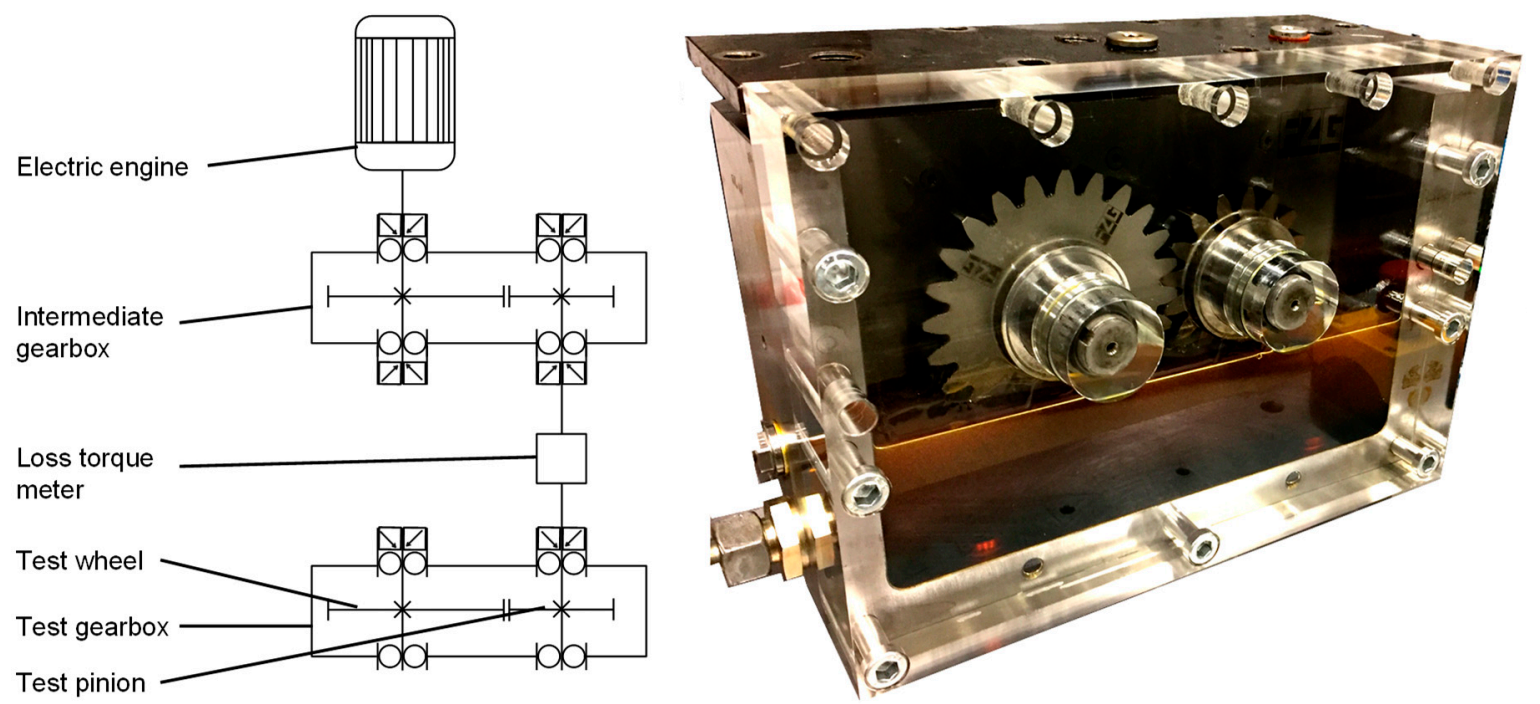

Figure 2. Mechanical layout of the FZG no-load power loss test rig (left) and the test gearbox with a transparent cover (right).

The speed of the electric engine is transmitted through the intermediate gearbox and the pinion shaft to the test gearbox. The no-load loss caused by the test gearbox is measured by the torque meter on the pinion shaft and includes the gear losses as well as the losses caused by the four bearings and two seals. In order to keep the losses from the bearings as small as possible, needle bearings are installed. The considered torque meter has a measuring range of $10 \mathrm{Nm}$ and a measuring error of $\pm 0.1 \%$. The oil sump temperature is monitored by a temperature sensor in the oil sump and controlled at the desired value with $\pm 2 \mathrm{~K}$. The test gearbox features a front cover made of transparent acryl glass in order to obtain a clear view on the oil flow. Thereby, a high-speed Photron FASTCAM Mini AX200 camera from VKT Video Kommunikation GmbH (Pfullingen, Germany) is used in front of the test gearbox to capture the oil flow with an image frequency of about 5000 frames per second.

\subsection{Test Gears}

As used by the authors in $[18,19]$, the considered test gears are of FZG type C-PT and made of case-carburized steel (16MnCr5E). The geometry data for both the pinion and wheel are shown in Table 1.

Table 1. Geometry of the type C-PT gears.

\begin{tabular}{|c|c|c|c|c|c|c|c|}
\hline Gear & a in $\mathrm{mm}$ & $\mathrm{z}_{1 \mid 2}$ & $m_{\mathrm{n}}$ in $\mathrm{mm}$ & $\alpha$ in deg & $x_{1 / 2}$ in $\mathrm{mm}$ & $b_{1 / 2}$ in $\mathrm{mm}$ & $\mathrm{d}_{\mathrm{a} 1 / 2}$ in $\mathrm{mm}$ \\
\hline Pinion (1) & 91.5 & 16 & 4.5 & 20.0 & 0.182 & 14 & 82.5 \\
\hline
\end{tabular}

\subsection{Lubricants}

Three mineral oils with considerably different viscosities are used. The mineral oil FVA3 with a viscosity grade of ISO VG 100 is used as a reference. In addition to this, the significantly more viscous oil FVA4 (ISO VG 460) and the low viscous oil FVA2 (ISO VG 32) are used as variant lubricants. 
The main properties of the considered lubricants are listed in Table 2. Note that FVA3 is the same base oil as considered by the authors in $[5,20]$.

Table 2. Properties of considered lubricants.

\begin{tabular}{|c|c|c|c|c|}
\hline Parameter & Symbol & FVA3 & FVA2 & FVA4 \\
\hline ISO VG & - & 100 & 32 & 460 \\
\hline Density at $40{ }^{\circ} \mathrm{C}$ in $\mathrm{kg} / \mathrm{m}^{3}$ & $\rho\left(15^{\circ} \mathrm{C}\right)$ & 864 & 855 & 882 \\
\hline Kinematic viscosity at $40^{\circ} \mathrm{C}$ in $\mathrm{mm}^{2} / \mathrm{s}$ & $v\left(40^{\circ} \mathrm{C}\right)$ & 95 & 32 & 480 \\
\hline Kinematic viscosity at $100{ }^{\circ} \mathrm{C}$ in $\mathrm{mm}^{2} / \mathrm{s}$ & $v\left(100^{\circ} \mathrm{C}\right)$ & 10.7 & 5.4 & 31.5 \\
\hline
\end{tabular}

\subsection{Operating Conditions}

Different circumferential speeds are investigated for the lubricants in Table 2. Each test series consists of six operating points per lubricant with the circumferential speed varying from $v_{t}=0.9 \mathrm{~m} / \mathrm{s}$ $\left(n_{1}=240 \mathrm{rpm}\right)$ to $v_{t}=20.0 \mathrm{~m} / \mathrm{s}\left(n_{1}=5225 \mathrm{rpm}\right)$. The low circumferential speeds are chosen to pronounce oil tracks at the pinion and the wheel for direct comparison of high-speed camera recordings with CFD simulation results. For higher circumferential speeds, the oil distribution gets too turbulent for a direct comparison. As, on the other hand, the churning losses cannot be resolved from measurements at the low circumferential speeds, the higher circumferential speeds are selected for additional comparison of measured churning losses with CFD simulation results. All measurements are carried out at an oil sump temperature of $40{ }^{\circ} \mathrm{C}$. The oil fill level is chosen so that $9 \mathrm{~mm}\left(2 \cdot m_{\mathrm{n}}\right)$ of the pinion dips into the oil sump. The operating conditions are summarized in Table 3.

Table 3. Considered operating conditions.

\begin{tabular}{|c|c|c|c|c|c|}
\hline $\begin{array}{l}\text { Pressure } \\
p \text { in Bar }\end{array}$ & Lubricant & $\begin{array}{c}\text { Oil Sump } \\
\text { Temperature } \\
\vartheta_{\text {Oil }}{ }^{\circ} \mathrm{C}\end{array}$ & Oil Fill Level in mm & $\begin{array}{l}\text { Rotational Speed of } \\
\text { Pinion } n_{1} \text { in rpm }\end{array}$ & $\begin{array}{l}\text { Circumferential } \\
\text { Speed at the Pitch } \\
\text { Circle } v_{t} \text { in } \mathrm{m} / \mathrm{s}\end{array}$ \\
\hline \multirow{6}{*}{1} & FVA3 & \multirow{6}{*}{40} & \multirow{6}{*}{$\begin{array}{l}32.2 \mathrm{~mm} \text { below the } \\
\text { middle axis (immersion } \\
\text { depth of } 9 \mathrm{~mm} \text { of pinion) }\end{array}$} & 240 & 0.9 \\
\hline & FVA2 & & & 360 & 1.4 \\
\hline & \multirow{4}{*}{ FVA4 } & & & 540 & 2.1 \\
\hline & & & & 2734 & 10.5 \\
\hline & & & & 4101 & 15.7 \\
\hline & & & & 5225 & 20.0 \\
\hline
\end{tabular}

The direction of rotation is chosen such that the wheel in Figure 2 rotates clockwise and the pinion, counter-clockwise.

\subsection{Derivation of the Gear Churning Loss}

The measured loss torque consists of no-load losses from the gear pair, the bearings and the seal in the test gearbox. In order to derive the hydraulic losses of the gears, two different test runs are conducted. The first test run is performed under dip-lubrication, whereas the second test run is performed after draining the oil from the test gearbox. As for the considered oil fill level (Table 3) the bearings and seal do not dip into the oil sump, their lubrication conditions are expected to be very comparable for both test runs. The hydraulic losses of the gear pair are determined by subtraction of the measured loss torques obtained from the first and second test runs. The hydraulic losses of the gear pair consist of the churning and the squeezing losses. As the squeezing loss can be, according to Mauz [2], neglected for the considered direction of rotation, the difference between the first and second test runs is interpreted as the churning loss of the gear pair. This procedure is expected to be more accurate than deriving the no-load gear loss by subtracting the calculated bearing and seal losses from the total loss torque as applied by the authors in $[5,20]$. Note that the gear drag torque is measured equally for the first and second test run and, hence, it is cancelled out. 


\section{Numerical Model}

The numerical model is implemented and solved in the commercial CFD software Ansys Fluent 18.0 (ANSYS, Inc., Canonsburg, PA, USA). As the model is a continuing work of Liu et al. [5], only the main physical and numerical characteristics and advancements are described.

\subsection{Governing Equations}

The governing equations consist of the conservation of mass, momentum and energy according to the Navier-Stokes equations. The energy conservation is not considered, as thermal influences can be neglected for no-load operating conditions.

During calculation, the conservation equations are solved on the finite volumes of the mesh model. In order to resolve the oil flow and the related flow properties, the grid size has to be in the same magnitude as the diameter of the oil droplets. When the rotational speed rises, the whole gearbox is filled with small oil droplets, which means that an extremely fine mesh has to be applied in order to resolve every single whirl. In this way, the computation time of turbulence flows can reach unacceptably high values as the number of equations rises cubically with the number of finite volumes.

To overcome this, turbulence models are introduced. These models do not resolve every single whirl, so a coarser mesh can be used, which requires much less computation time. In these equations, the fluid flow quantities in the conservation equations are separated into a time-weighted average term and an oscillating term. The oscillating term is determined by transport equations, so that the averaged Navier-Stokes equations can be written in closed form. In this study, a k- $\varepsilon$ model consisting of two coupled transport equations is used.

Further information on the governing equations can be found in the previous work of the authors in [5]. The $\mathrm{k}-\varepsilon$ model used in this study represents a good compromise between computation time, result quality, and stability $[1,18]$. Further analyses have not shown noteworthy differences to the $\mathrm{k}-\varepsilon$ model.

The fluid is considered as a Newtonian fluid. Thus, the shear stress is linearly proportional to the shear rate. The dynamic viscosity is constant irrespective of the shear rate and only varies as a function of temperature according to the data provided in Table 3. Surface roughness and surface tension are not considered in the simulation model.

\subsection{Geometry and Mesh}

The conservation equations are solved iteratively on finite volumes during calculation. The simulation model based on hexahedral elements presented by the authors in [5] has been adjusted to a simulation model based on tetrahedral elements. Pre-investigations have shown that this simulation model provides better predictions of the oil flow, as the mesh deforming only affects the elements within the gear moving zone.

The mesh model of the single-stage test gearbox of the FZG no-load power loss test rig in Figure 2 essentially consists of four domains: the pinion and wheel domain, the gearbox domain, and the domain of the remeshing zone. The employed simulation model has already been described by the authors in [5]. In order to improve the readability, its main structure is repeated and supplemented by modifications compared to [5] in the following. The entire model represents a negative model of the test gearbox. The mesh of each single domain is connected with the other domains as shown in Figure 3. The mesh of the pinion and wheel domain is discretized with inflation layers and does not undergo any mesh deformation. During the simulation, the meshes of the pinion and wheel domain rotate inside the gearbox domain at predefined circumferential speeds. The gearbox domain consists of tetrahedral elements and also does not undergo any mesh deformation. The domain of the remeshing zone fills the cavity between the gearbox domain and the pinion and wheel domain. During operation, the meshing zone of the pinion and wheel is a transient area that changes at every gear meshing position. Therefore, the remeshing zone consists of a deformable meshing structure that 
changes with every time step of the rotating pinion and wheel domain. The domain of the remeshing zone is discretized with deformable tetrahedral elements, which are geometrically flexible and follow every rigid movement of the gears. In comparison to the mesh model applied in the previous work in [5], the mesh model of this study consists of prisms and tetrahedral elements.
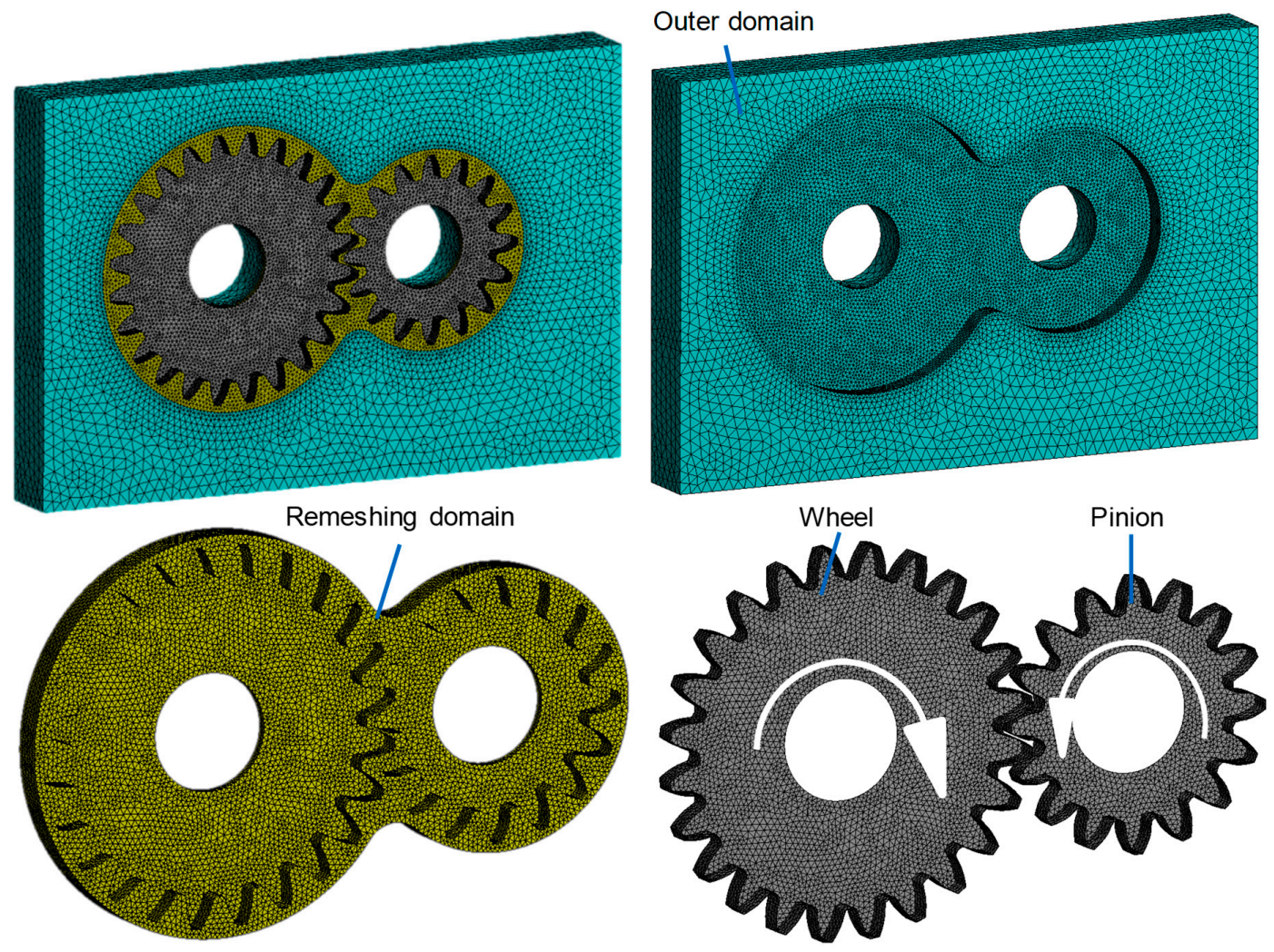

Figure 3. Entire mesh model (top left); outer domain of the gearbox (top right); remeshing domain (bottom left); and pinion and wheel domain (bottom right).

In order to avoid poor element quality and numerical singularities in the very small gap between the tooth flanks, i.e., backlash, both the pinion and the wheel are scaled to $98 \%$ of their actual size. This is currently required for all FV-based CFD simulations of meshing gears. The scaling is not expected to significantly influence the oil distribution and churning loss. Mauz [22] shows that losses caused by squeezing the oil particles between tooth flanks can be neglected for the considered direction of rotation.

In the mesh model, symmetry boundaries are used to reduce the extent of the computational model to a symmetric subsection. Thus, the middle plane of the test gearbox was set to the "symmetry" boundary condition. On this plane, the flux is zero and the normal velocity, as well as the normal gradients of all fluid variables, are assumed to be zero. The boundary walls of the outer domain, which represents the walls of the test gearbox, were set to "wall" boundary conditions, which assures the velocity of all fluids to be zero.

For the simulation series, 40 cores of a high-performance computing cluster were used as the hardware. In order to predict the oil flow for direct comparison with high-speed camera recordings in detail, a very fine grid with $2.3 \mathrm{M}$ elements was applied to the mesh model, which results in an element size smaller than $1 \mathrm{~mm}^{3}$. The time step size was set to $0.1^{\circ}$ rotation of the pinion. A local convergence criterion of $10^{-5}$ was used for all equations. These settings result in a calculation time of 12-15 $\mathrm{h}$ for a single rotation of the pinion. Note that the described simulation model is customized for the purpose of this study resulting in this comparable high calculation time. 
Grid sensibility analyses of the numerical model were performed by varying the element number from $0.2 \mathrm{M}$ to $2.3 \mathrm{M}$. The primary object of the investigation was the churning loss torque of the pinion and wheel. The maximum deviation of the churning loss torques was only about $4 \%$, however, the oil flow could be resolved best with the highest element number.

\subsection{Derivation of the Gear Churning Loss}

Resistant torques are derived by multiplying the pressure on each finite volume of the tooth flanks with the associated area and the lever arm to the center of the pinion and wheel, respectively. Subsequent summation of all resistant torques provides the overall gear no-load loss torque. This value consists of the churning, squeezing, and the windage loss portion. The windage loss can be derived from an additional single phase simulation with only air as a medium. As the squeezing loss can be, according to Mauz [2], neglected for the considered direction of rotation, the churning loss torque of the gear pair is derived by subtracting the resulting windage loss torque from the overall loss torque.

\section{Results}

The main focus of this study is the oil distribution. In addition, the churning loss is considered. A total of 12 operating points is investigated. CFD simulation results are compared with high-speed camera recordings for low circumferential speeds and with loss torque measurements for higher circumferential speeds. Furthermore, simulated velocity and oil fraction plots are considered for evaluation of the physical plausibility of the simulation results.

\subsection{Oil Distribution}

In order to check the capability of the considered FV-based CFD simulation model for detailed prediction of the oil flow in gearboxes, very low circumferential speeds are considered resulting in characteristic oil tracks behind both the pinion and the wheel. The nine considered operating conditions are defined by the circumferential speeds $v_{t}$ of $0.9 \mathrm{~m} / \mathrm{s}, 1.4 \mathrm{~m} / \mathrm{s}$, and $2.1 \mathrm{~m} / \mathrm{s}$, and the lubricants FVA2, FVA3, and FVA4. The numerical results are compared with high-speed recordings of the test gearbox of the FZG no-load power loss test rig. Figure 4 shows the obtained oil distributions by the CFD simulation model and the high-speed camera.

After the start-up of the engine, the oil is gradually taken out of the oil sump and carried along with the teeth, until it reaches the meshing zone, in which it is squeezed from the narrowing tooth gap. As the circumferential speed increases, the oil dragged along the tooth flanks is gradually swung off the teeth forming visible oil tracks starting at the tooth tips. When the prescribed circumferential speed is reached, the form of the oil tracks finally takes its characteristic shape.

It can clearly be seen that the oil flows reveal major differences in a comparison among the considered mineral oils. Hence, the high-speed camera recordings clearly highlight the difference in oil viscosity. Due to the small differences in oil density between FVA2, FVA3, and FVA4 (cf. Table 2), its influence is expected to be subordinate.

When the reference oil FVA3 is used, the centrifugal force swings the oil off the tooth flanks and leaves characteristic oil tracks in the upper half of the gearbox. On the pinion side, the approximately 1.5-fold centrifugal force compared to the wheel is able to swing a greater amount of oil off the tooth flanks, so that pronounced oil tracks fill the right half of the gearbox. On the wheel side, due the weaker centrifugal force, the oil swung off the tooth flanks does not reach the space range of the oil thrown off by the pinion. When the circumferential speed is increased to $v_{t}=1.4 \mathrm{~m} / \mathrm{s}$, the centrifugal force is approximately 2.3 -fold compared to $v_{t}=0.9 \mathrm{~m} / \mathrm{s}$. This leads to more expanded oil tracks in space as the oil is thrown a further distance from the gears. When the speed continues to rise to $v_{t}=2.1 \mathrm{~m} / \mathrm{s}$, a larger number of small oil droplets fills the area above the mating zone. These smaller oil droplets are mainly formed by the collision of droplets. On the one hand, oil drips from the top cover, which was dragged there by the rotating gears. On the other hand, the oil tracks left behind by both the pinion and wheel collide with each other at the area above the mating zone. 

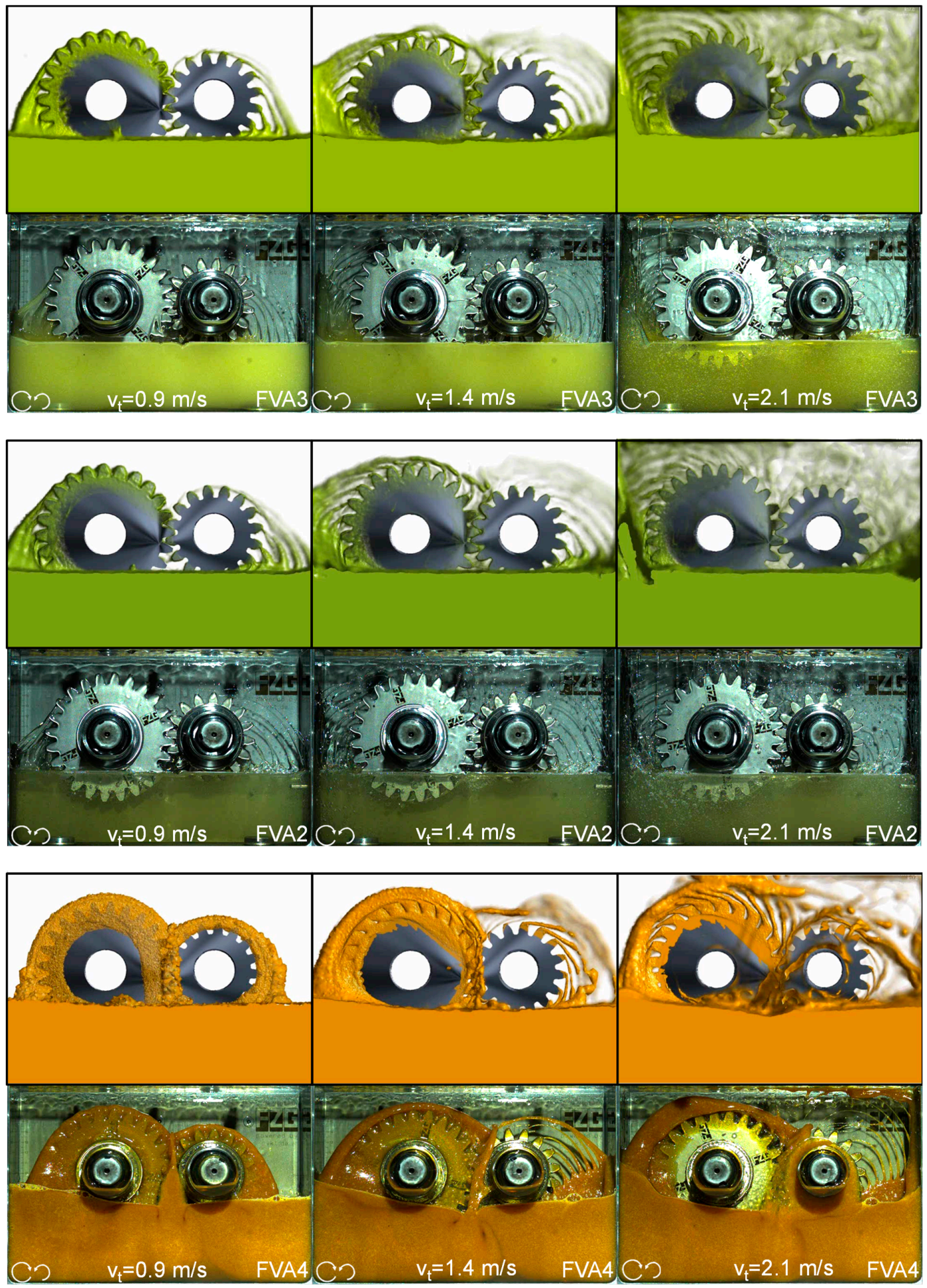

Figure 4. Simulated oil distribution in comparison with high-speed camera recordings for circumferential speeds of $v_{t}=\{0.9 ; 1.4 ; 2.1\} \mathrm{m} / \mathrm{s}$ for FVA3, FVA2, and FVA4 $\left(\vartheta_{\text {oil }}=40^{\circ} \mathrm{C}\right)$.

In order to analyze the influence of the viscosity, the less viscous mineral oil FVA2 is used. In general, the oil flow is similar to FVA3. However, due to the lower level of resistance to shear stress of FVA2, the oil particles are thrown a further distance. Furthermore, the formed oil tracks are thinner than for FVA3. Instead of refined oil tracks on both sides as observed for FVA3, the oil tends to turn 
into irregular larger oil droplets on the side of the wheel, as well as at the region above the mating zone, particularly when the circumferential speed is increased to $v_{t}=1.4 \mathrm{~m} / \mathrm{s}$ and $v_{t}=2.1 \mathrm{~m} / \mathrm{s}$.

When the far more viscous lubricant FVA4 is used, the oil flow appears completely different. The resistance to shear stress of FVA4 is higher compared to FVA3 and FVA2. Thus, especially when the circumferential speed is only $v_{t}=0.9 \mathrm{~m} / \mathrm{s}$, the oil dragged by gears "sticks" together and is not swung away. Hence, a sort of belt is formed around both gears instead of refined oil tracks. When the circumferential speed is increased to $v_{t}=1.4 \mathrm{~m} / \mathrm{s}$, the higher centrifugal force at the tooth tips of the pinion results in higher momentum of the oil particles, which is great enough to form refined oil tracks instead of oil belts. For the circumferential speed of $v_{t}=2.1 \mathrm{~m} / \mathrm{s}$, the centrifugal force rises and a larger "belt" is formed. Furthermore, the oil tracks left by the pinion are thrown a further distance, so that some of them reach the top cover of the gearbox. As compared to FVA3 and FVA2, less oil is swung off from the tooth flanks along the way to the gear mating zone, a higher amount of oil is dragged into the gear meshing zone and squeezed out. It can also clearly be observed that a greater amount of oil is present on the front surface of the gears. This is also due to the high resistance to shear stress of FVA4. Another notable aspect is the dynamic deformation of the oil sump, which differs significantly from FVA3 and FVA2. Especially on the side of the wheel, the oil sump is deflected to a considerable angle.

The simulation results for the oil flow in Figure 4 are generally in very good agreement with the high-speed recordings. The influence of the circumferential speed and the lubricant viscosity is captured well and even the characteristic oil tracks are present in the simulation results. Only the resolution of the oil tracks and of small dispersed oil droplets (especially for FVA2), as well as the results with FVA4 leave room for improvement.

One advantage of three-dimensional CFD simulations is that the oil flow behavior in the gearbox can be evaluated from arbitrary perspectives for different quantities. In the following, the oil fraction and velocity distribution is supplemented for the operating conditions in Figure 4. Thereby, the front and side view in the middle planes of the test gearbox are considered. This provides detailed insights into the oil flow behavior.

The oil fraction is shown in Figure 5. It can be seen that the oil sump under the meshing zone is partly dug free. For FVA3 and FVA2, the tooth gaps of teeth in the oil sump are filled with a comparatively small portion of air with the oil being displaced to predominately one side of the tooth gaps. Due to the higher oil viscosity and the resulting lower oil flow velocity of FVA4, the tooth gaps of teeth in the oil sump are almost fully filled with air, whereas the oil has no time to flow in the tooth gaps before the teeth exit the oil sump. This effect is more pronounced with increasing speed and influences the churning loss torque. The side view of the gear meshing zone shows squeezing of oil in the axial direction. In general, the higher the circumferential speed is, the weaker the squeezing effect in axial direction. This is due to the increasing centrifugal forces spinning the oil off from the tooth flanks before reaching the gear meshing zone. As expected, FVA4 shows the largest oil squeezing in the axial direction, especially at low circumferential speed. As a result of its high resistance to shear stress, which keeps the oil sticking together on the tooth flanks, forming a belt as shown in Figure 4, the lubricant is almost fully dragged into the gear mating zone, in which a lot of it is squeezed in the axial direction.

The velocity distribution is shown in Figure 6. According to the Stokes adhesive condition, the velocities of the Newtonian fluid particles on the surfaces of the pinion and wheel are equal to the corresponding circumferential speed $v_{t}$. The oil velocity outside the tip circle gradually decreases as the distance from the center of the pinion and wheel increases. The fluid particles in the tooth gaps have similar velocity as the gear flanks. Turbulence with higher velocities can occur in the transitional zone. When comparing the velocity distribution between FVA2, FVA3, and FVA4, it can be noticed that it correlates well with the oil flow field shown in Figure 4. In general, the less viscous the lubricant is, the smaller and faster the oil particles become, which leads to a more scattered velocity field. Therefore, just like the oil flow field shown in Figure 4, FVA2 and FVA3 give a similar velocity distribution field, while FVA4 shows a less scattered velocity field. 

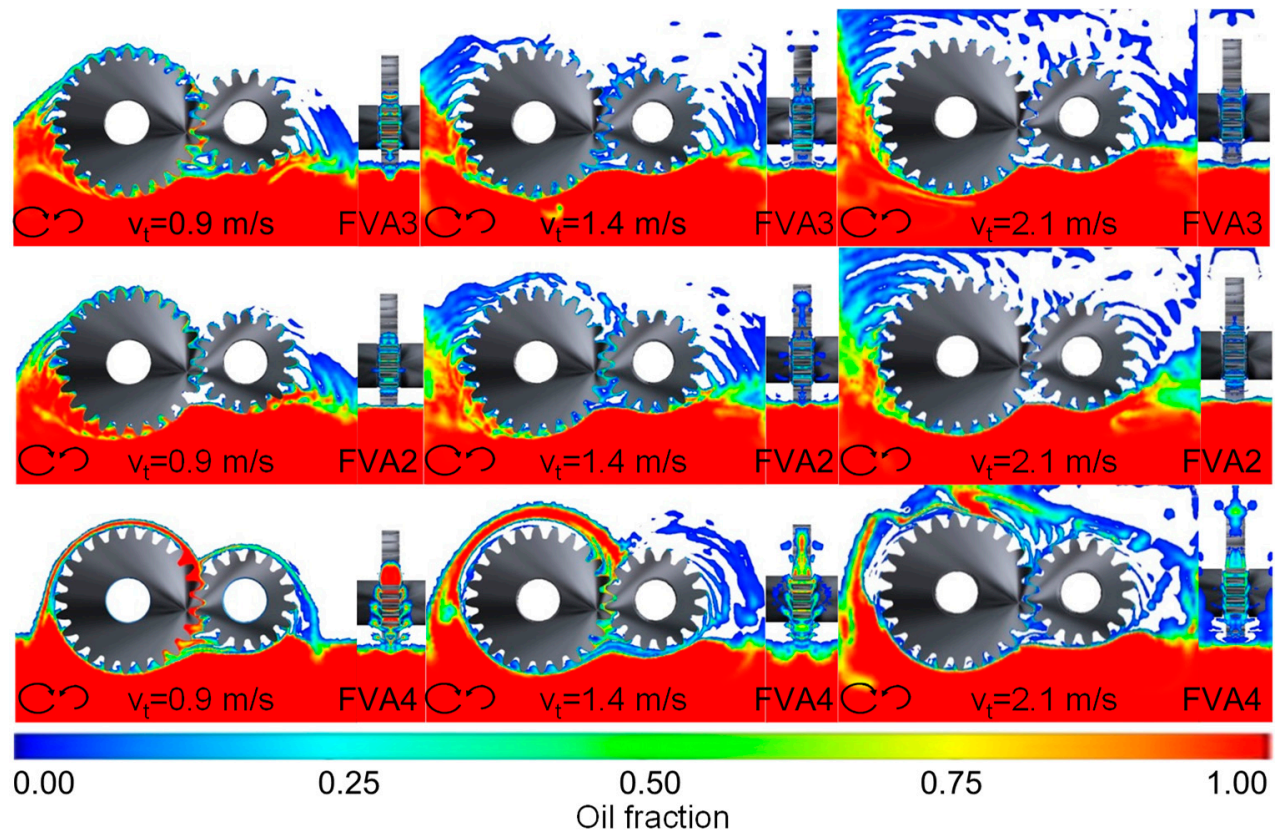

Figure 5. Simulated oil fraction from the front and side view in the middle planes of the test gearbox for circumferential speeds of $v_{t}=\{0.9 ; 1.4 ; 2.1\} \mathrm{m} / \mathrm{s}$ for mineral oil FVA3, FVA2, and FVA4 $\left(\vartheta_{\text {oil }}=40{ }^{\circ} \mathrm{C}\right)$.

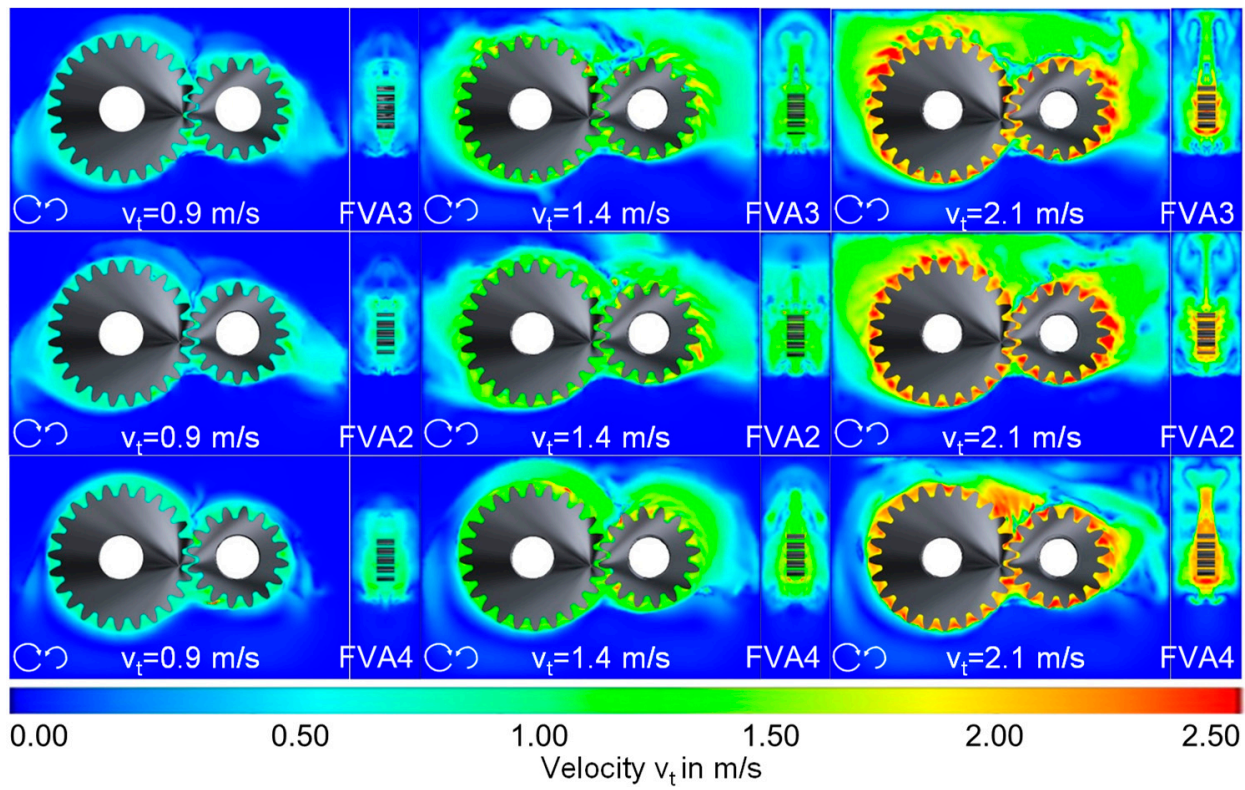

Figure 6. Simulated velocity distribution from the front and side view in the middle planes of the test gearbox for circumferential speeds of $v_{t}=\{0.9 ; 1.4 ; 2.1\} \mathrm{m} / \mathrm{s}$ for mineral oil FVA3, FVA2, and FVA4 $\left(\vartheta_{\text {oil }}=40^{\circ} \mathrm{C}\right)$.

\subsection{Churning Power Loss}

Supplementary to the investigation of oil distribution, the churning loss of the gear pair is investigated for the mineral oil FVA3. Higher circumferential speeds $v_{t}$ of $10.5 \mathrm{~m} / \mathrm{s}, 15.7 \mathrm{~m} / \mathrm{s}$ and $20.0 \mathrm{~m} / \mathrm{s}$ are considered, because the very low churning losses cannot be resolved from measurements for the low circumferential speeds of Section 4.1.

Figure 7 shows the corresponding CFD simulation and measurement results. The simulated churning losses are obtained according to Section 3.2. Note that the subtracted windage power losses 
share only up to $5 \%$ of the overall gear no-load power losses. The measured churning power losses are obtained according to Section 2.5. For each measured operating point, the mean value and its standard deviation are determined based on ten measurements for the first and second test run, respectively. The measurement uncertainty propagation, according to [9], is then used to obtain the shown mean and standard deviation.

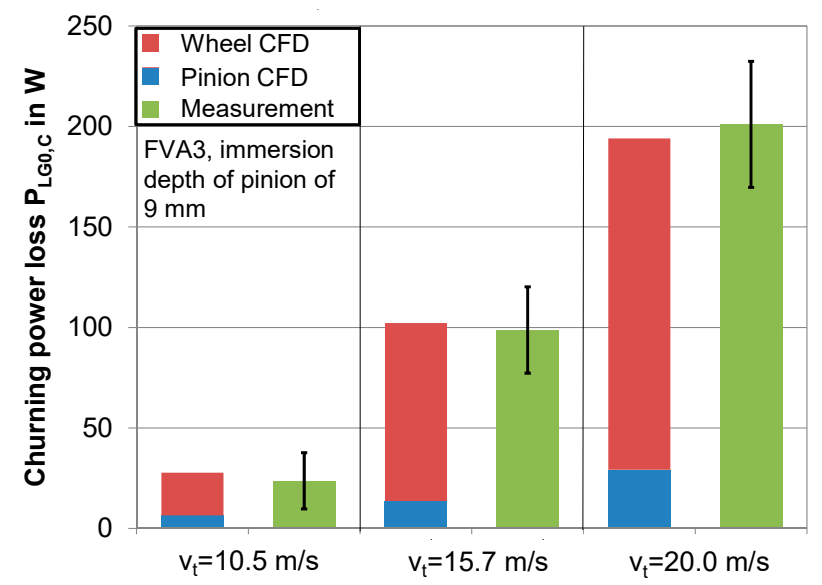

Figure 7. Comparison of gear churning power loss between CFD simulation and measurement for circumferential speeds of $v_{t}=\{10.5 ; 15.7 ; 20.0\} \mathrm{m} / \mathrm{s}$ for mineral oil FVA3 $\left(\vartheta_{\text {oil }}=40^{\circ} \mathrm{C}\right)$.

The simulated gear churning losses in Figure 7 are in very good agreement with the measured ones. Both show an increasing trend with increasing circumferential speeds. As shown in a previous study [19], the turbulence in the oil sump increases resulting in a gradual oil filling of the empty space of the gearbox. This results in higher churning loss torques with increasing circumferential speeds.

By means of CFD simulation, the churning power loss caused by the pinion and wheel can be evaluated separately. Figure 7 shows that the pinion only shares a percentage of $13-23 \%$ of the total churning losses. This has two main reasons: on the one hand, the pinion dips less into the oil sump compared to the wheel, so that there is much less surface on which the oil can offer resistance; on the other hand, due to the higher centrifugal force compared to the wheel, the oil sump under the pinion is more significantly dug free especially at high circumferential speeds when the oil has no time to flow back to the tooth gaps before the teeth exit the oil sump.

\section{Conclusions}

In this work, a finite volume CFD simulation model was applied to analyze the oil flow of a single-stage gearbox of the FZG no-load power loss gear test rig. This test rig was specially designed for the measurement of oil distribution and no-load loss with high accuracy. The simulated oil distributions and their comparison with high-speed camera recordings showed that the influence of both oil viscosity and circumferential speed can be captured very well. Even detailed behaviors of the oil flow can be identified by the simulation results. Additional oil fraction and velocity distribution plots from different perspectives showed the plausibility of the results. It was also shown that, based on the CFD simulation model, churning loss portions caused by both pinion and wheel can easily be evaluated separately for meshing gears. Future work will focus on different influencing factors, including lubrication, geometry, and operating conditions, in order to make use of CFD to improve the oil flow and efficiency of gearboxes.

Supplementary Materials: Supplementary Materials are available online at http:/ www.mdpi.com/2075-4442/ 6/2/47/s1.

Author Contributions: H.L. performed the simulations, analyzed the results and wrote the paper. T.J. consulted regarding the design of the simulations and participated in the scientific discussions. T.L. additionally revised the paper. K.S. supported the interpretation of the results and proof read the paper. 
Acknowledgments: This work was supported by the German Research Foundation (DFG) and the Technical University of Munich (TUM) in the framework of the Open Access Publishing Program.

Conflicts of Interest: The authors declare no conflict of interest.

\section{References}

1. Concli, F. Efficiency of Gear Transmissions and CFD Analysis of the Load Independent Power Losses. Ph.D. Thesis, Politecnico di Milano, Milan, Italy, 2012.

2. Mauz, W. Hydraulische Verluste von Stirnradgetrieben bei Umfangsgeschwindigkeiten bis $60 \mathrm{~m} / \mathrm{s}$. Ph.D. Thesis, Universität Stuttgart, Stuttgart, Germany, 1987.

3. Strasser, D. Einfluss des Zahnflanken- und Zahnkopfspiels auf die Leerlaufverlustleistung von Zahnradgetrieben. Ph.D. Thesis, Universität Bochum, Bochum, Germany, 2005.

4. Walter, P.; Langenbeck, K.; Dittrich, O. FVA-Nr. 44/I. Heft 118 Anwendungsgrenzen für die Tauchschmierung von Zahnradgetrieben, Plansch- und Quetschverluste bei Tauchschmierung; FVA: Frankfurt/Main, Germany, 1982.

5. Liu, H.; Jurkschat, T.; Lohner, T.; Stahl, K. Determination of oil distribution and churning power loss of gearboxes by finite volume CFD method. Tribol. Int. 2017, 109, 346-354. [CrossRef]

6. Doleschel, A. Wirkungsgradberechnung von Zahnradgetrieben in Abhängigkeit vom Schmierstoff. Ph.D. Thesis, Technical University of Munich, Munich, Germany, 2002.

7. Leprince, G.; Changenet, C.; Ville, F.; Velex, P.; Jarnias, F. Influence of oil aeration on churning losses. In Proceedings of the JSME International Conference on Motion and Power Transmissions, Sendai, Japan, 13-15 May 2009. [CrossRef]

8. Otto, H.-P. Flank Load Carrying Capacity and Power Loss Reduction by Minimised Lubrication. Ph.D. Thesis, Technical University of Munich, Munich, Germany, 2009.

9. Li, L.; Versteeg, K.; Hargrave, G.K.; Potter, T.; Halse, C. Numerical Investigation on Fluid Flow of Gear Lubrication. SAE Int. J. Fuels Lubr. 2009, 1, 1056-1062. [CrossRef]

10. Jafarian, P. Gear interlocking effect study using CFD. In Proceedings of the 3rd International CTI Symposium “Automotive Transmissions, HEV \& EV Drives", Berlin, Germany, 8-11 December 2014.

11. Klier, C.; Berger, L. New prospects for oil flow simulation in rotating spur-gear systems. In Proceedings of the International Conference on Gears 2015, Garching b. München, Germany, 5-7 October 2015.

12. Concli, F. Low-loss gears for precision planetary gearboxes. Influence of the gear design on the meshing and the churning power losses. In Proceedings of the International Conference on Gears 2015, Garching b. München, Germany, 5-7 October 2015. [CrossRef]

13. Concli, F. Gearbox efficiency: The role of CFD. In Summer School Summer School 2016; Politecnico di Milano, Technical University of Munich: Milan, Italy, 2016.

14. Concli, F. Numerical modelling of the churning power losses of gears: An innovative 3D computational tool suitable for planetary gearbox simulation. Tribol. Int. 2016, 103, 58-68. [CrossRef]

15. Concli, F.; Gorla, C. CFD Simulations of windage losses of a gearbox. In Proceedings of the International CAE Conference 2012, Verona, Italy, 22-23 October 2012.

16. Concli, F.; Gorla, C. Numerical modeling of the power losses in geared transmissions: Windage, churing and cavitation simulations with a new integrated approach that drastically reduces the computational effort. Tribol. Int. 2016, 103, 58-68. [CrossRef]

17. Burberi, E.; Fondelli, T.; Andreini, A.; Facchini, B.; Cipolla, L. CFD Simulations of a Meshing Gear Pair. In Proceedings of the ASME Turbo Expo 2016: Turbomachinery Technical Conference and Exposition, Seoul, Korea, 13-17 June 2016.

18. Gorla, C.; Concli, F.; Stahl, K.; Höhn, B.-R.; Michaelis, K.; Schultheiß, H.; Stemplinger, J.-P. CFD Simulations of Splash Losses of a Gearbox. Adv. Tribol. 2012, 2012, 616923. [CrossRef]

19. Lucy, L.B. A numerical approach to the testing of the fission hypothesis. Astron. J. 1977, 82, $1013-1024$. [CrossRef]

20. Liu, H.; Arfaoui, G.; Stanic, M.; Montigny, L.; Jurkschat, T.; Lohner, T.; Stahl, K. Numerical Modelling of Oil Distribution and Churning Gear Power Losses of Gearboxes by Smoothed Particle Hydrodynamics. J. Eng. Tribol. 2017. [CrossRef] 
21. Iino, T.; Matsumura, S.; Houjoh, H.; Hachiya, T. Calculation of the behavior of oil churning and its loss in a gear box by a moving particle method-The first result of both calculation and experiment. In Proceedings of the International Conference on Power Transmissions 2016, Chongqing, China, 16-20 October 2016.

22. Groenenboom, P.H.L.; Mettichi, M.Z.; Gargouri, Y. Simulating Oil Flow for Gearbox Lubrication using Smoothed Particle Hydrodynamics. In Proceedings of the International Conference on Gears 2015, Garching b. München, Germany, 5-7 October 2015.

23. Liu, H.; Jurkschat, T.; Lohner, T.; Stahl, K. Numerical modeling and validation of oil distribution and churning losses in gearboxes. In Proceedings of the 6th World Tribology Congress 2017, Beijing, China, 17-22 September 2017.

C 2018 by the authors. Licensee MDPI, Basel, Switzerland. This article is an open access article distributed under the terms and conditions of the Creative Commons Attribution (CC BY) license (http://creativecommons.org/licenses/by/4.0/). 